hep-th/9604140

BONN-TH-96-02

IFP-607-UNC

April 1996

\title{
Exploring the Moduli Space of $(0,2)$ Strings
}

\author{
Ralph Blumenhagen ${ }^{1}$ and Andreas Wißkirchen ${ }^{2}$ \\ ${ }^{1}$ Institute of Field Physics, Department of Physics and Astronomy, \\ University of North Carolina, Chapel Hill NC 27599-3255, USA \\ 2 Physikalisches Institut der Universität Bonn, Nußallee 12, 53115 Bonn, Germany
}

\begin{abstract}
We use an exactly solvable $(0,2)$ supersymmetric conformal field theory with gauge group $S O(10)$ to investigate the superpotential of the corresponding classical string vacuum. We provide evidence that the rational point lies in the Landau-Ginzburg phase of the linear $\sigma$-model and calculate exactly all three- and four-point functions of the gauge singlets. These couplings already put severe constraints on the possible flat directions of the superpotential. Finally, we contemplate about the flat direction related to Kähler deformations of the underlying linear $\sigma$-model.
\end{abstract}

1 e-mail: blumenha@physics.unc.edu

2 e-mail: wisskirc@avzw02.physik.uni-bonn.de 


\section{Introduction}

Mainly during the last three years progress has been made in showing that the class of $(2,2)$ supersymmetric string compactifications is only a small subset of all four-dimensional perturbative heterotic string vacua featuring $N=1$ space-time supersymmetry $[2-5,11,13-$ $15,21,26,27,29]$. It was long believed that the general class of $(0,2)$ strings might not be solutions of the string equations of motion at all, for these models could receive destabilizing instanton corrections [10,28]. However, in a paper by E. Silverstein and E. Witten [27] it was argued that for the class of linear $\sigma$-models such terms in the superpotential cannot occur due to the absence of singularities in the singlet couplings. Shortly afterwards in [4] we constructed heterotic exactly solvable $(0,2)$ superconformal field theories (SCFTs) exhibiting all the properties required for $(0,2)$ string vacua. For instance, the phenomenologically most interesting feature is that the gauge group is not restricted to $E_{6}$ as in the $(2,2)$ case [7] but can also be $S O(10)$ or $S U(5)$ [28]. The main difficulty turned out to really identify SCFTs with special points in the moduli space of a Calabi-Yau $\sigma$-model with a choice of a stable, holomorphic vector bundle for the left moving $\sigma$-model fermions. In [5] for at least three $N=1$ models such an identification has been shown to be possible, including the $S O(10)$ model we will focus on in this paper. Moreover, for the class of $N=2$ space-time supersymmetric strings it was furthermore possible to identify all constructed SCFTs with certain bundles on $K_{3} \times T_{2}$ [6].

In this paper we will investigate the moduli space of a concrete $(0,2)$ model by for the first time calculating all three- and four-point functions of the gauge singlets at the exactly solvable point. In [14] using the Landau-Ginzburg description of the quintic in $\mathbb{C P}[4]$ it was already shown that besides the well known $(2,2)$ moduli space [8] containing the complex and Kähler deformations there are flat directions in the superpotential due to elements of $H^{1}(\operatorname{End}(T))$, as well. At least all states coming from untwisted sectors of the LG orbifold are mutually integrable. In [27] it was argued, and exemplified again for the case of the quintic, that even more is true. All deformations related to parameters of the linear $\sigma$-model are moduli for any value of the Kähler class. At the Landau-Ginzburg point this includes some moduli from twisted sectors. Thus, for the quintic in $\mathbb{C P}[4]$ there is a 326 -dimensional $(0,2)$ moduli space containing a 102 -dimensional $(2,2)$ subspace. One advantage of the knowledge of an exactly solvable model for a $(0,2)$ string vacuum is that it allows one to make definite statements about nonvanishing superpotential couplings, in general at least to finite order in the superpotential. We study in detail the $(0,2)$ model with gauge group $S O(10)$ which was first constructed in [4] and then related to the CalabiYau manifold $\mathbb{P}_{1,1,1,1,2,2}[44]$ with the bundle $V(1,1,1,1,1 ; 5)$ in [5]. After identifying our SCFT with a special point in the Landau-Ginzburg sector, we calculate the couplings of all singlets to fourth order and find that they do not all vanish. At the Landau-Ginzburg point there occur more than the 329 massless singlets expected from the large radius limit. Thus, not all singlets at the Landau-Ginzburg point correspond to flat directions of the space-time superpotential. Unlike the $(2,2)$ case $[12,17]$, at small radius there exists no algebraic distinction among the complex, Kähler and bundle moduli. However, in our special model, requiring certain properties expected for the Kähler modulus and using Fflatness and D-flatness for the special enhanced gauge symmetry, at least to lowest order the possible candidates for the deformation of the radius are highly restricted. 
This paper is organized as follows. In section 2 . we review the construction of the $(0,2)$ SCFT. In section 3. we give evidence that the SCFT lies in the Landau-Ginzburg phase of the corresponding linear $\sigma$-model. In section 4 . we make use of the SCFT to calculate all holomorphic three- and four-point functions of the $S O(10)$ singlets. Finally, in section 5. we use these results to restrict the form of the Kähler modulus and to speculate about new flat directions in the superpotential not belonging to the moduli space of the linear $\sigma-$ model.

\section{The exactly solvable model}

To begin with, we briefly review the model we will deal with in this paper. The details of the construction of general $(0,2)$ SCFTs can be found in $[4,5]$. In order to achieve heterotic modular invariant partition functions we made use of the technique of simple currents $[19,22-25]$. In light cone gauge the starting point of the construction is the tensor product of CFTs as shown in Table 2.1.

\begin{tabular}{|l|l|l|}
\hline part & $c$ & $\bar{c}$ \\
\hline $4 D$ space-time, $X^{\mu}$ & 2 & 2 \\
\hline$N=2$ Virasoro & 9 & 9 \\
\hline$U(1)_{2}$ & 1 & 1 \\
\hline gauge group $S O(8) \times E_{8}$ & 12 & 12 \\
\hline
\end{tabular}

Table $2.1 \quad$ Underlying CFT for $S O(10)$

Introducing a special set of simple currents guaranteeing all properties we want to have for $(0,2)$ models like left moving $N=2$ world sheet supersymmetry, projection onto even $U(1)$ charges and an extension of the gauge group from $S O(8)$ to $S O(10)$, we obtain modular invariant partition functions of the following form:

$$
Z \sim \vec{\chi}(\tau) M\left(J_{G S O_{l}}\right) \prod_{j} M\left(\Upsilon_{j}\right) M\left(J_{G S O_{r}}\right) \prod_{i} M\left(J_{i}\right) M\left(J_{(S O(8) \rightarrow S O(10))}\right) \vec{\chi}(\bar{\tau}) .
$$

In order to really get $(0,2)$ models one has to choose some model dependent simple currents $\Upsilon_{j}$, which prevent all the symmetries implemented on the right moving side from acting also on the left moving side. In the model discussed in [5], the internal $N=2$ part consists of five copies of the $k=3$ minimal $N=2$ model $(\mathrm{N} 2 \operatorname{Vir}(\mathrm{k}=3))$ and $\Upsilon$ is chosen to be

$$
\Upsilon=\Phi_{0,-1}^{3} \otimes\left(\Phi_{0,0}^{0}\right)^{4} \otimes \Phi_{1,2}^{U(1)_{2}} \otimes \Phi_{0}^{S O(8)},
$$

where $\Phi_{m, s}^{l}$ denotes the highest weight representations of the $k=3$ minimal model. Thus $\Upsilon$ acts nontrivially only on the first factor of the internal tensor product and on the $U(1)_{2}$ part. The massless spectrum contains the usual $N=1$ supergravity multiplet, chiral multiplets in the four possible representations of $S O(10)_{1}$ and also some vector multiplets. There are precisely $N_{16}=80$ chiral fields in the spinor representation, $N_{10}=74$ chiral fields in the vector representation and $N_{1}=350$ chiral fields in the singlet representation of $S O(10)$. Furthermore, besides the gauge bosons of $S O(10) \times E_{8}$ the spectrum contains 
$N_{g}=7$ further vector multiplets. Since the simple current $\Upsilon$ itself and its charge conjugate are two of these seven further gauge bosons, the special enhanced gauge group cannot simply be $U(1)^{7}$. It is easy to see that the three fields of conformal dimension one

$$
\begin{aligned}
J^{ \pm}(z) & =\Phi_{\mp 1}^{3}(z) \otimes e^{\mp i \sqrt{\frac{5}{3}} \phi_{1}(z)} \otimes e^{ \pm i \frac{1}{2} \phi_{U(1)_{2}}(z)}, \\
J^{3}(z) & =\frac{1}{2}\left(-5 j_{1}(z)+3 j_{U(1)_{2}}(z)\right)
\end{aligned}
$$

satisfy the $S U(2)$ Kac-Moody algebra at level $k=3 . \Phi_{\mp 1}^{3}(z)$ are primary fields of the $k=3$ parafermionic model and the $U(1)$ currents of the first $\mathrm{N} 2 \mathrm{Vir}(\mathrm{k}=3)$ model and the $U(1)_{2}$ model are $j_{1}=i \sqrt{\frac{3}{5}} \partial \phi_{1}$ and $j_{U(1)_{2}}=i \partial \phi_{U(1)_{2}}$, respectively. Thus, the complete gauge group of this model is $G=S O(10) \times E_{8} \times S U(2)_{3} \times U(1)^{4}$ and the massless spectrum should also fit into the four allowed representations of $S U(2)_{3}$.

In [5] we have listed the explicit form of the massless states in the spinor and vector representation of $S O(10)$ and have given a monomial representation of these states such that the Yukawa couplings $\left\langle\begin{array}{llll}10 & 16 & 16\end{array}\right.$ could be written as a monomial ring $\mathbb{C}\left[x_{i}, y_{j}\right] / I$. The $x_{i}$ are four coordinates of weight one and the $y_{j}$ are two coordinates of weight two. The ideal $I$ is generated by the relations $x_{i}^{4}=0$ and $y_{j} y_{k}=0$. The same monomial ring appears as the cohomological ring of the Calabi-Yau manifold $\mathbb{P}_{1,1,1,1,2,2}[44]$ with the gauge bundle $V(1,1,1,1,1 ; 5)$. Thus, we concluded that the exactly solvable SCFT describes a certain point in the moduli space of this $(0,2)$ model. However, we have not determined to which point the SCFT corresponds, namely what the form is of the quasihomogeneous polynomials $W_{1,2}\left(x_{i}, y_{j}\right)$ and $F_{1, \ldots, 5}\left(x_{i}, y_{j}\right)$ defining the complete intersection $\mathrm{CY}$ and the vector bundle $V$, respectively. Furthermore, one has to know at which radius $r$ of the Kähler modulus the model lives. In order to answer these questions we also have to take the singlet fields into account.

A singlet in the $(-1)$ ghost picture is of the general form

$$
V_{-1}(z, \bar{z})=e^{-\rho(\bar{z})} \mathcal{O}_{1}(z, \bar{z}) \mathcal{F}(z) e^{i k X(z, \bar{z})},
$$

where $\mathcal{O}_{1}$ is an internal operator of the $N=2$ theory with $(c, \bar{c})=(9,9)$ and $\mathcal{F}$ denotes the left moving $U(1)_{2}$ part. The product $\mathcal{O}_{1} \mathcal{F}$ has overall conformal dimension $(h, \bar{h})=\left(1, \frac{1}{2}\right)$ and charge $(q, \bar{q})=(0,-1)$. In Table 2.2 and 2.3 we list the explicit form of these internal operators for all the 350 singlets occurring in the model. The degeneracy is due to three reasons. Firstly, there is the $S_{4}$ permutation symmetry of the last four N2Vir $(\mathrm{k}=3)$ tensor factors. Secondly, we have the four allowed $S U(2)_{3}$ representations with degenerated ground states of dimension one to four. In Table 2.2 and 2.3 we always list the state with highest value of the $U(1)_{2}$ quantum number. Thus, the other states in the $S U(2)$ multiplet can be obtained by applying successively $J^{-}$. Finally, whenever a state like, for instance ${ }^{1}$

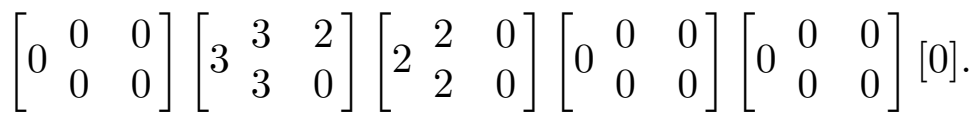

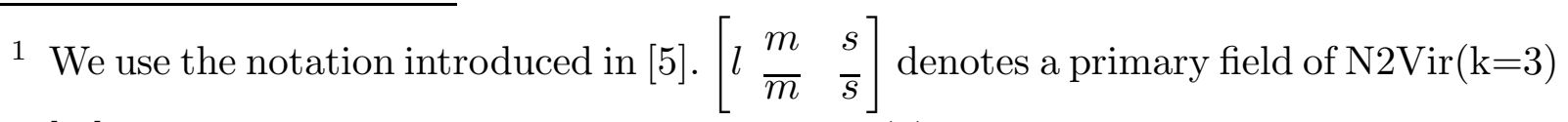
and $[m]$ denotes one of the four primary fields of $U(1)_{2}$. 
occurs there is also a state

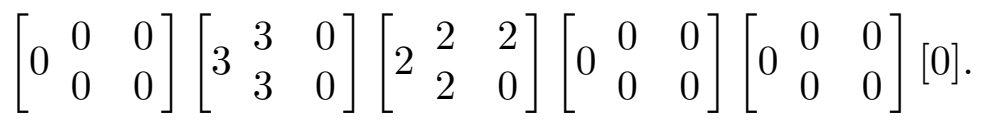

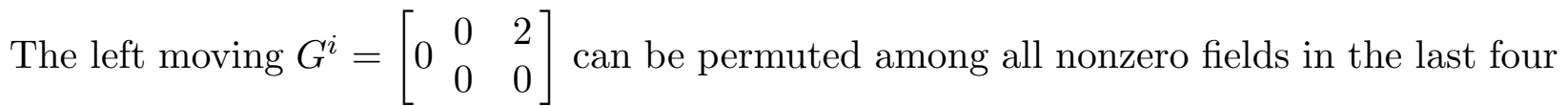
$N=2$ tensor factors. In accordance to [14] we denote the untwisted fields by $S$ and the twisted ones by $S^{\prime}$.

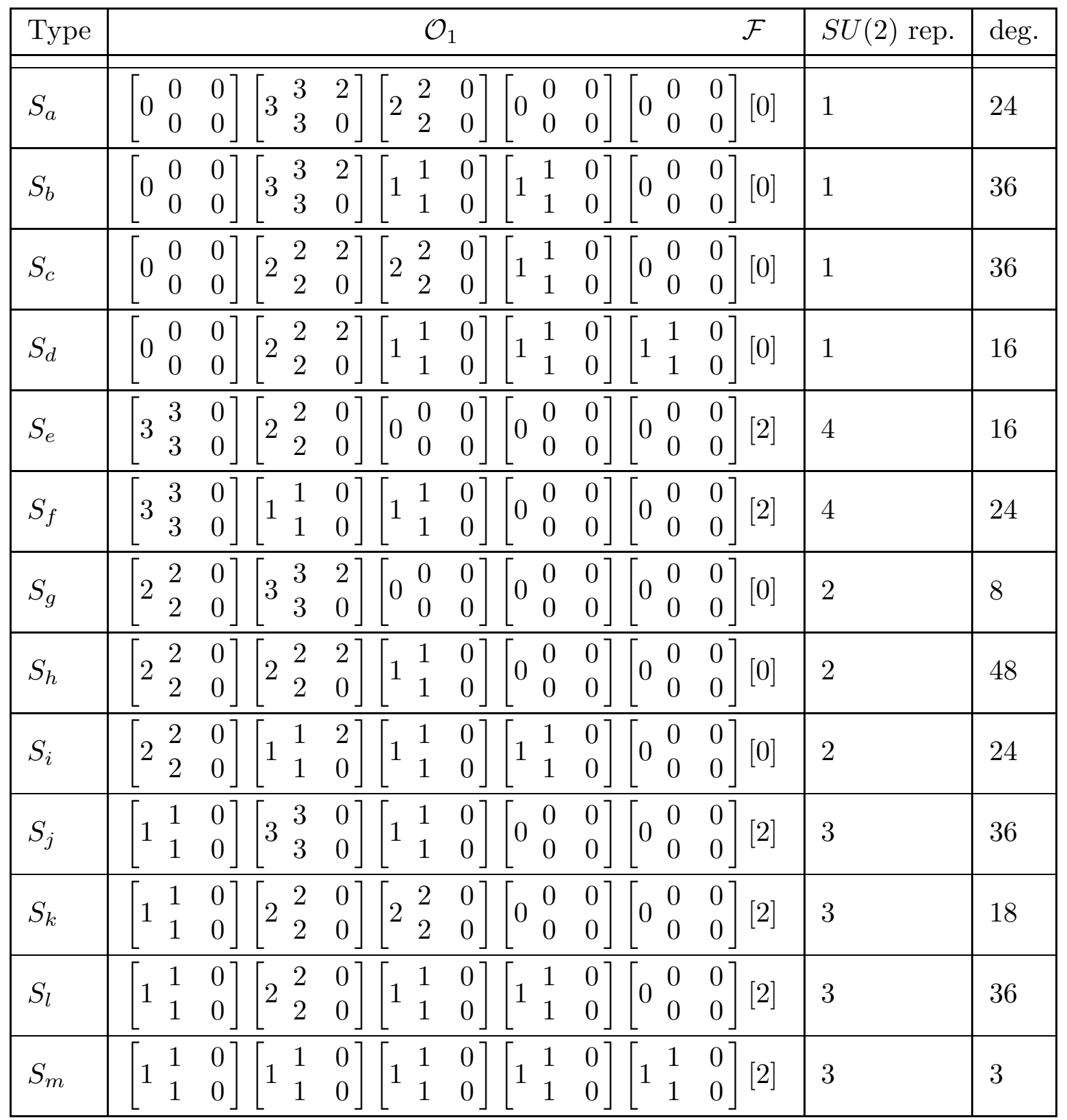

Table 2.2 Untwisted Singlets

These are exactly 325 of the 350 singlets. The remaining 25 states occur in twisted sectors of the $G S O_{r}$ projection. 


\begin{tabular}{|l|l|c|l|l|}
\hline Type & $\mathcal{O}_{1}$ & $\mathcal{F}$ & rep. & deg. \\
\hline$T^{\prime}$ & {$\left[\begin{array}{lll}1 & 1 \\
0 & 0\end{array}\right]\left[\begin{array}{ccc}2 & -2 & 0 \\
2 & 0\end{array}\right]\left[\begin{array}{ccc}1 & -3 & -2 \\
1 & 0\end{array}\right]\left[\begin{array}{cc}-3 & -2 \\
1 & 0\end{array}\right]\left[\begin{array}{cc}-3 & -2 \\
1 & 0\end{array}\right][1]$} & 1 & 4 \\
\hline$S_{a}^{\prime}$ & {$\left[\begin{array}{ccc}3 & -4 & -1 \\
3 & 0\end{array}\right]\left[\begin{array}{ccc}1 & -1 & 0 \\
1 & 0\end{array}\right]\left[\begin{array}{ccc}1 & -1 & 0 \\
1 & 0\end{array}\right]\left[\begin{array}{cc}-2 & -2 \\
0 & 0\end{array}\right]\left[\begin{array}{cc}-2 & -2 \\
0 & 0\end{array}\right][1]$} & 1 & 6 \\
\hline$S_{b}^{\prime}$ & {$\left[\begin{array}{ccc}2 & 5 & 5 \\
2 & 0\end{array}\right]\left[\begin{array}{ccc}1 & -1 & 0 \\
1 & 0\end{array}\right]\left[\begin{array}{ccc}1 & -1 & 0 \\
1 & 0\end{array}\right]\left[\begin{array}{ccc}1 & -1 & 0 \\
1 & 0\end{array}\right]\left[\begin{array}{cc}0 & -2 \\
0 & 0\end{array}\right][1]$} & 3 & 12 \\
\hline$S_{c}^{\prime}$ & {$\left[\begin{array}{ccc}1 & -1 & -2 \\
1 & 0\end{array}\right]\left[\begin{array}{ccc}1 & -1 & 0 \\
1 & 0\end{array}\right]\left[\begin{array}{ccc}1 & -1 & 0 \\
1 & 0\end{array}\right]\left[\begin{array}{ccc}1 & -1 & 0 \\
1 & 0\end{array}\right]\left[\begin{array}{ccc}1 & -1 & 0 \\
1 & 0\end{array}\right][0]$} & 3 & 3 \\
\hline
\end{tabular}

Table 2.3 Twisted Singlets

These numbers of untwisted and twisted singlets have to be compared with the numbers of singlets in the LG phase of the corresponding linear $\sigma$-model. It has been shown in [13] that for a generic choice of the $W_{i}(x, y)$ and $F_{i}(x, y)$ the model contains 339 singlets, 318 of which are untwisted. Note that in the Calabi-Yau limit the number of singlets is only

$$
H^{1}(M, T)+H^{1}\left(M, T^{*}\right)+H^{1}(M, \operatorname{End}(V))=73+1+255=329 .
$$

\section{The Landau-Ginzburg phase}

In order to identify the SCFT with the Landau-Ginzburg phase of the linear $\sigma$-model we have at least to show that the massless spectra are the same. For our model of interest the $(0,2)$ superpotential is

$$
S_{\mathcal{W}}=\int d^{2} z d \theta\left(\Sigma_{j} W_{j}(X, Y)+\Lambda_{a} F_{a}(X, Y)\right)
$$

where $\Sigma_{1,2}$ and $\Lambda_{1, \ldots, 5}$ are Fermi superfields and $X_{1, \ldots, 4}$ and $Y_{1,2}$ are chiral superfields. $W_{1,2}(X, Y)$ and $F_{1, \ldots, 5}(X, Y)$ are quasihomogenous polynomials of degree four. In the Landau-Ginzburg phase there exists a right $U(1) \mathrm{R}$-symmetry with charges $\bar{q}$ and a left $U(1)$ symmetry with charges $q$. In Table 3.1 we list all the charges of the fields involved in the calculation of the massless spectrum.

\begin{tabular}{|l|l|l|}
\hline field & $q$ & $\bar{q}$ \\
\hline$x_{1, \ldots, 4}$ & $\frac{1}{5}$ & $\frac{1}{5}$ \\
\hline$y_{1,2}$ & $\frac{2}{5}$ & $\frac{2}{5}$ \\
\hline$\sigma_{1,2}$ & $-\frac{4}{5}$ & $\frac{1}{5}$ \\
\hline$\lambda_{1, \ldots, 5}$ & $-\frac{4}{5}$ & $\frac{1}{5}$ \\
\hline
\end{tabular}

\begin{tabular}{|l|l|l|}
\hline field & $q$ & $\bar{q}$ \\
\hline $\bar{x}_{1, \ldots, 4}$ & $-\frac{1}{5}$ & $-\frac{1}{5}$ \\
\hline $\bar{y}_{1,2}$ & $-\frac{2}{5}$ & $-\frac{2}{5}$ \\
\hline $\bar{\sigma}_{1,2}$ & $\frac{4}{5}$ & $-\frac{1}{5}$ \\
\hline $\bar{\lambda}_{1, \ldots, 5}$ & $\frac{4}{5}$ & $-\frac{1}{5}$ \\
\hline
\end{tabular}

Table 3.1 Left and right charges

For generic choices of the polynomials the massless spectrum has been calculated for the Landau-Ginzburg orbifold in [13]. There are 80 chiral superfields in the spinor representation, 74 superfields in the vector representation and 339 superfields in the singlet representation of $\mathrm{SO}(10)$. The question is, whether there exists a choice of the polynomials $W_{j}$ 
and $F_{a}$ such that there occur 350 singlets, 325 untwisted and 25 twisted, accompanied by an enhanced gauge group of dimension seven. In [5] we have already made the following guess for the form of the constraints:

$$
\begin{aligned}
W_{1}(X, Y) & =\sum_{i=1}^{4} X_{i}^{4}+\sum_{j=1}^{2} Y_{j}^{2}, & W_{2}(X, Y) & =\sum_{i=1}^{4} i X_{i}^{4}+\sum_{j=1}^{2} j Y_{j}^{2} \\
F_{i}(X, Y) & =X_{i}^{4} \quad \text { for } i \in\{1, \ldots, 4\}, & F_{5}(X, Y) & =Y_{1} Y_{2},
\end{aligned}
$$

which was motivated by the fact that the exactly solvable model has the permutation symmetry $S_{4}$ in the last four $N=2$ tensor factors. In the Landau-Ginzburg model the $U(1)$ gauge symmetry of the linear $\sigma$-model is spontaneously broken to a finite group $\mathbb{Z}_{5}$, so that one actually deals with an orbifold theory. Furthermore, to get a heterotic string theory one has to combine the internal Landau-Ginzburg sector with the linear part of the gauge group, which is $S O(8)$ in our case. The GSO projection then selects states with $g=1$ for

$$
g=\exp \left(-i \pi J_{0}\right) \times(-1)^{\lambda} .
$$

$J_{0}$ is the left $U(1)$ charge and $(-1)^{\lambda}$ denotes the charges of the different $S O(8)$ representations. The resulting orbifold has sectors twisted by $g^{k}$ for $k=0, \ldots, 9$. If $k$ is even we call them $(R, R)$ sectors and if $k$ is odd we call them $(N S, R)$ sector. Finally, since one is only interested in the massless sector of the string model, one can employ a Born-Oppenheimer approximation and truncate the fields to their lowest excited modes. The right moving $N=2$ algebra

$$
\left\{\bar{Q}_{-}, \bar{Q}_{+}\right\}=\bar{L}_{0}, \quad \bar{Q}_{-}^{2}=\bar{Q}_{+}^{2}=0
$$

tells us that massless states are given by the cohomology of $\bar{Q}_{+}$. There is an expression even off-criticality for this operator in terms of the fundamental fields in the Lagrangian:

$$
\bar{Q}_{+}=i \int\left(i \bar{\psi}^{i} \partial \phi_{i}+\left.\mathcal{W}\right|_{\theta=0}\right)
$$

By splitting this into $\bar{Q}_{+}=\bar{Q}_{+, r}+\bar{Q}_{+, l}$ it was shown in [20] that one can simply calculate the cohomology of $\bar{Q}_{+, l}$ in the cohomology of $\bar{Q}_{+, r}$. In order to get more insight into these methods the interested reader may take a look into $[13,20]$. By going through the calculation of the massless spectrum carried out in [13], one realizes that there are really more states for the choice of the polynomials in (3.2). Table 8 of [13] shows that the massless singlets get modified in the way described in Table 3.2.

\begin{tabular}{|l|l|l|}
\hline$S O(8) \times U(1)$ & $k$ & State \\
\hline $1_{0}$ & 1 & $P_{4}\left(\Phi_{-\frac{q_{i}}{2}}^{i}\right) \lambda_{-\frac{3}{5}}^{a}|0\rangle, P_{4}\left(\Phi_{-\frac{q_{i}}{2}}^{i}\right) \sigma_{-\frac{3}{5}}^{j}|0\rangle$ \\
\hline $1_{0}$ & 3 & $\lambda_{-\frac{1}{5}}^{a} \lambda_{-\frac{1}{5}}^{b}|0\rangle, \lambda_{-\frac{1}{5}}^{a} \sigma_{-\frac{1}{5}}^{j}|0\rangle, \sigma_{-\frac{1}{5}}^{1} \sigma_{-\frac{1}{5}}^{2}|0\rangle$ \\
\hline $1_{0}$ & 5 & $\bar{\lambda}_{0}^{a} \bar{\lambda}_{0}^{b} \bar{\lambda}_{0}^{c}|0\rangle$ for $a, b, c \in\{1, \ldots, 4\}$ \\
\hline
\end{tabular}

Table 3.2 Massless LG singlets 
$P_{4}\left(\Phi_{-\frac{q_{i}}{2}}^{i}\right)$ denotes a polynomial of weight four in the six coordinates $x_{i}, y_{j}$. The untwisted states for $k=1$ have to be considered modulo the equivalence relations

$$
\begin{aligned}
& F_{a}\left(\Phi_{-\frac{q_{i}}{2}}^{i}\right) \lambda_{-\frac{3}{5}}^{a}|0\rangle \sim F_{a}\left(\Phi_{-\frac{q_{i}}{2}}^{i}\right) \sigma_{-\frac{3}{5}}^{j}|0\rangle \sim 0 \\
& W_{j}\left(\Phi_{-\frac{q_{i}}{2}}^{i}\right) \lambda_{-\frac{3}{5}}^{a}|0\rangle \sim W_{j}\left(\Phi_{-\frac{q_{i}}{2}}^{i}\right) \sigma_{-\frac{3}{5}}^{j}|0\rangle \sim 0 \\
& P_{2}\left(\Phi_{-\frac{q_{i}}{2}}^{i}\right)\left[\frac{\partial F_{a}}{\partial y_{-\frac{1}{5}}^{1,2}} \lambda_{-\frac{3}{5}}^{a}+\frac{\partial W_{j}}{\partial y_{-\frac{1}{5}}^{1,2}} \sigma_{-\frac{3}{5}}^{j}\right]|0\rangle \sim 0 \\
& P_{1}\left(\Phi_{-\frac{q_{i}}{2}}^{i}\right)\left[\frac{\partial F_{a}}{\partial x_{-\frac{1}{10}}^{1, \ldots, 4}} \lambda_{-\frac{3}{5}}^{a}+\frac{\partial W_{j}}{\partial x_{-\frac{1}{10}}^{1, \ldots, 4}} \sigma_{-\frac{3}{5}}^{j}\right]|0\rangle \sim 0 .
\end{aligned}
$$

For generic $W_{j}$ and $F_{a}$ there are 318 such states. However, for the symmetric choice in (3.2) we obtain 325 states, which are inevitably accompanied by seven further gauge fields. Furthermore, there occur four singlet fields from the $k=5$ sector, which are not present at a generic point in the moduli space. Thus, together with the 21 states from the $k=3$ twisted sector there are 25 twisted singlets, the same number as for the exactly solvable model. This brief excursion to $(0,2)$ Landau-Ginzburg models has provided some more evidence that we can identify the exactly solvable model with a $(0,2)$ Landau-Ginzburg model naturally appearing in the $r \rightarrow-\infty$ limit of a linear $\sigma$-model. In the following section we will calculate all three- and four-point functions of the SCFT.

\section{Singlet couplings in the superpotential}

Unlike, for instance, the quintic and the corresponding Landau-Ginzburg model, in our $(0,2)$ case the number of $S O(10)$ singlets in the large radius limit is different from the number of singlets in the Landau-Ginzburg phase. Since we are now also equipped with an explicit SCFT description, it is possible to investigate moduli in the neighbourhood of the exactly solvable point. Thus, we are looking for integrable marginal deformations of the SCFT preserving the right moving $N=2$ world sheet supersymmetry. From the spacetime point of view this is equivalent to searching for flat directions in the four-dimensional effective low energy $N=1$ space-time supersymmetric field theory. The scalar potential for such supergravity theories is generally known as [1,9]

$$
U=e^{\mathcal{K}}\left(D^{i} W G_{i j^{*}}^{-1} D^{j^{*}} W^{*}-3 W\right)+\frac{1}{2} \sum_{a}\left(D^{a}\right)^{2},
$$

where $\mathcal{K}\left(\phi_{i}, \phi_{i}^{*}\right)$ is the Kähler potential, $W\left(\phi_{i}\right)$ the holomorphic superpotential and $T^{a}$ generators of the gauge group. The covariant derivative is given by

$$
D^{i} W=\frac{\partial W}{\partial \phi_{i}}+\frac{\partial \mathcal{K}}{\partial \phi_{i}} W
$$

and $D^{a}$ are auxiliary fields in the vector multiplets of the gauge bosons

$$
D^{a}=-\frac{g_{a}}{2}\left(\frac{\partial \mathcal{K}}{\partial \phi_{i}} T^{a} \phi_{i}+\phi_{i}^{*} T^{a} \frac{\partial \mathcal{K}}{\partial \phi_{i}^{*}}\right) .
$$


In the lowest order, the renormalizable field theory limit, $\mathcal{K}$ is flat and the scalar potential takes the form

$$
U=\sum_{a} \frac{g_{a}^{2}}{2}\left|D^{a}\right|^{2}+\sum_{i}\left|F_{i}\right|^{2}=\sum_{a} \frac{g_{a}^{2}}{2}\left(\phi_{i}^{*} T^{a} \phi_{i}\right)^{2}+\sum_{i}\left|\frac{\partial W}{\partial \phi_{i}}\right|^{2}
$$

where $W$ contains only cubic couplings. In order for the scalar potential to vanish both the $\mathrm{D}$-terms and the $\mathrm{F}$-terms have to be zero.

For gauge singlets the condition of D-flatness is satisfied automatically and one has only to check $\mathrm{F}$-flatness for the superpotential. However, even though we are dealing mostly with $S O(10)$ singlets we have to cope with the D-terms arising from the enhanced gauge symmetry $S U(2) \times U(1)^{4}$. The superpotential of the low energy effective field theory can be determined in the SCFT by calculating correlation functions of the corresponding vertex operators on the $S^{2}$ world sheet. For the following discussion one has to have in mind that the vertex operators in the SCFT geometrically are tangent vectors along the moduli space. Suppose one finds a set of scalars $\left\{S_{i}\right\}$, such that all D-terms vanish and all superpotential couplings of the form $F(S)$ and $F(S) S^{\prime}$ are zero for all scalars $S^{\prime}$ in the model. Then the entire set $\left\{S_{i}\right\}$ are flat directions and define bona fide moduli of the theory. However, if one has a set of scalars for which not all terms in the scalar potential vanish, then one has to be very careful in drawing any conclusions. It does not necessarily mean that there are no flat directions at all. This can be seen by studying the following well known example: Consider the simple case of a complex boson with a global $U(1)$ symmetry and the Higgs potential

$$
V(\phi)=\lambda\left(|\phi|^{2}-\frac{m^{2}}{2 \lambda}\right)^{2}
$$

Now, expanding around one minimum $\langle\phi\rangle=v=\sqrt{\frac{m^{2}}{2 \lambda}}$ in the usual way

$$
\phi=\eta+v+i \chi
$$

one finds in the potential

$$
V(\phi)=\lambda\left(4 v^{2} \eta^{2}+4 v \eta\left(\eta^{2}+\chi^{2}\right)+\left(\eta^{2}+\chi^{2}\right)^{2}\right)
$$

both $\eta^{2}$ and $\chi^{4}$ couplings. Thus, neither of the two fields satisfy $F(S)=0$. Nevertheless, we know that there is a flat direction, the circle with radius $v$. By looking at the lowest order term $\eta^{2}$ one can read off that this flat direction locally is $(\eta, \chi)=\varepsilon(0,1)$. Surely, choosing polar coordinates the angular variable does not appear in the potential and defines the flat circle. In our case however, the local coordinates are given by the primary fields in the SCFT and without further knowledge there is no guarantee that these are appropriate to capture certain flat directions explicitly. Furthermore, unlike this model, one generally does not know the superpotential to all orders, so that extracting definite statements is a difficult task. In the next section we will exactly be confronted with such problems, when we try to identify the Kähler modulus. 
First, we want to discuss the former sufficient flatness condition in our example. In general it is hard to prove in CFT that an infinite set of $n$-point functions vanishes unless one is equipped with some selection rules which a priori disallow certain couplings to be nonzero. In our case we have a lot of such selection rules related to the special enhanced gauge symmetry. On the one hand side both the left and the right moving $U(1)$ symmetries from each of the $\mathrm{N} 2 \operatorname{Vir}(\mathrm{k}=3)$ and $U(1)_{2}$ factors have to be preserved. On the other hand side there is the special nonabelian $S U(2)$ gauge symmetry, which also constraints the possible couplings. In particular, the $S U(2)$ spins have to couple to zero spin in each correlation function and the relative couplings of members of $S U(2)$ multiplets are determined by Clebsch-Gordan coefficients.

To make the discussion more transparent we introduce something like an average, relative charge between the left and right moving sectors of the singlets. All the singlets in the Tables 2.2 and 2.3 share the common feature that in each of the last four $\mathrm{N} 2 \operatorname{Vir}(\mathrm{k}=3)$ factors five times the difference between the left and the right moving $U(1)$ charge $q_{\text {rel }}$ is constant modulo five. For instance, for the field $T^{\prime}$

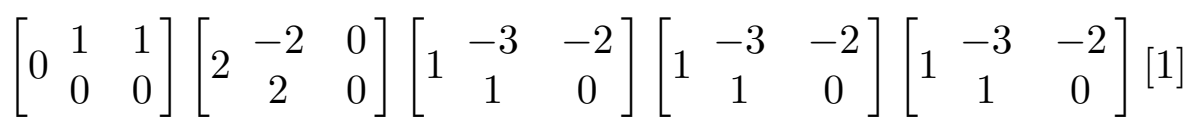

one has

$$
Q\left(T^{\prime}\right)=5\left(\left(\frac{3}{5}-1\right)-\left(-\frac{1}{5}\right)\right)=5\left(\left(\frac{2}{5}\right)-\left(-\frac{2}{5}\right)\right)=4 \bmod 5 .
$$

One can also extend this definition to the other massless states in the $\mathbf{1 6}$ and $\mathbf{1 0}$ representation. In order to make it well-defined one has to take into account the charge of the $S O(8)$ piece, as well. Considering the decomposition of representations of $S O(10)$ in terms of $S O(8) \times U(1)$

$$
[16]=\left[8_{v}^{1}\right] \oplus\left[8_{c}^{-1}\right], \quad[10]=\left[1^{-2}\right] \oplus\left[8_{s}^{0}\right] \oplus\left[1^{2}\right]
$$

the correct definition is

$$
Q=5 q_{\mathrm{rel}}-\frac{1}{2}(-1)^{\lambda} \bmod 5 .
$$

This definition is very similar to the space-time $\mathrm{R}$-charge $S_{Q}$ introduced in $[14,15]$ for the massless states in the Landau-Ginzburg model, for it contains information about the twisted sector of the GSO projection, in which the massless states occur. One obtains for all the massless states in the model Table 4.1 of space-time $\mathrm{R}$-charges:

\begin{tabular}{|l|l|l|l|l|l|}
\hline$S$ & $T^{\prime}$ & $S^{\prime}$ & $\mathbf{1 6}$ & $\mathbf{1 0}$ & $\mathbf{1 0}^{\prime}$ \\
\hline 0 & 4 & 2 & $-\frac{1}{2}$ & -1 & 3 \\
\hline
\end{tabular}

Table 4.1 R-charges of massless states

Note that this charges are completely analogous to the R-charges of the different massless fields in the Landau-Ginzburg model, thus providing further evidence for the identification 
of these two models. The general form of a superpotential coupling of order $n$ is expressed in terms of world sheet operators in the following way:

$$
C^{1, \ldots, n}=\int d^{2} z_{n} \ldots \int d^{2} z_{1}\left\langle V_{0}^{n}\left(z_{n}, \bar{z}_{n}\right) \ldots V_{0}^{4}\left(z_{4}, \bar{z}_{4}\right) V_{-1}^{3}\left(z_{3}, \bar{z}_{3}\right) V_{-\frac{1}{2}}^{2}\left(z_{2}, \bar{z}_{2}\right) V_{-\frac{1}{2}}^{1}\left(z_{1}, \bar{z}_{1}\right)\right\rangle \text {. }
$$

The lower index indicates the ghost picture, in which the vertex operator has to be taken. Using the $S L(2, \mathbb{C})$ symmetry one can shift in the usual way three coordinates to $\{0,1, \infty\}$ and can get rid of three integrations by including the correct measure, for instance

$$
\int d^{2} z_{n} \ldots \int d^{2} z_{4}\left|z_{1}-z_{2}\right|^{2}\left|z_{1}-z_{3}\right|^{2}\left|z_{2}-z_{3}\right|^{2} \ldots
$$

If the vertex operator for a massless state in a general representation of the gauge group has the form

$$
V_{-1}(z, \bar{z})=e^{-\rho(\bar{z})} \mathcal{O}_{1}(z, \bar{z}) \mathcal{F}(z) \lambda^{a}(z) e^{i k X(z, \bar{z})}
$$

in the $(-1)$ ghost picture, in the $\left(-\frac{1}{2}\right)$ ghost picture it will look like

$$
V_{-\frac{1}{2}}(z, \bar{z})=e^{-\frac{\rho(\bar{z})}{2}} S^{\alpha}(\bar{z}) \Sigma_{r} \mathcal{O}_{1}(z, \bar{z}) \mathcal{F}(z) \lambda^{a}(z) e^{i k X(z, \bar{z})}
$$

with $S^{\alpha}(\bar{z})$ being a four-dimensional spinor and $\Sigma_{r}$ the internal right moving part of the space-time supercharge. In our case, it is simply the primary field

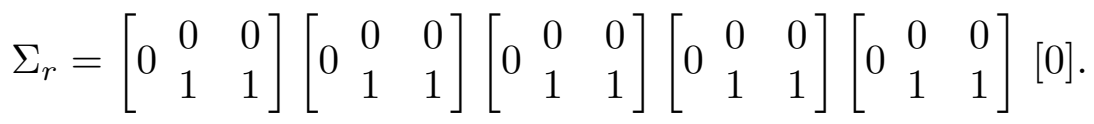

In the (0) ghost picture one gets

$$
V_{0}(z, \bar{z})=\left(G_{r, \text { tot }}+k \psi\right) \mathcal{O}_{1}(z, \bar{z}) \mathcal{F}(z) \lambda^{a}(z) e^{i k X(z, \bar{z})}
$$

where $G_{r, \text { tot }}$ is the total world sheet supercurrent of the $c=9$ right moving $N=2$ part:

$$
G_{r, \text { tot }}=\sum_{i=1}^{5}\left[\begin{array}{lll}
0 & 0 & 0 \\
& 0 & 0
\end{array}\right] \cdots \underbrace{\left[\begin{array}{lll}
0 & 0 & 0 \\
& 0 & 2
\end{array}\right]}_{i \text { th factor }} \cdots\left[\begin{array}{lll}
0 & 0 & 0 \\
0 & 0 & 0
\end{array}\right][0] .
$$

One can also attach an R-charge to the two operators $\Sigma_{r}$ and $G_{r \text {,tot }}$ appearing in the $-\frac{1}{2}$ and 0 ghost picture, respectively. Since $Q\left(\Sigma_{r}\right)=-\frac{3}{2}$ and $Q\left(G_{r, \text { tot }}\right)=0$, the sum of all $Q$ charges of all internal fields $\mathcal{O} \mathcal{F}$ must be equal to $Q=3$ in order have vanishing $\mathrm{R}$-charge for the entire coupling constant. Thus, formally a term in the superpotential must have $Q=3$ yielding already severe constraints on the possible terms in the superpotential. Surely, Yukawa couplings like $\left\langle\begin{array}{llll}10 & 16 & 16\end{array}\right.$ or $\langle S \quad 1010\rangle$ could take nonzero values but couplings like $\left\langle 10^{\prime} 1616\right\rangle$ or $\left\langle S^{\prime} 1010\right\rangle$ are forced to be zero by $Q$ conservation. For instance, among the couplings of twisted fields only $\left\langle S^{\prime} 10^{\prime} 10^{\prime}\right\rangle$ can also be nonzero. We 
will come back to such couplings later. Since all untwisted singlets have zero R-charge, it follows directly that

$$
F(S)=0, \quad F(S) S^{\prime}=0 .
$$

However, one must not forget the seven D-flatness conditions for $S U(2) \times U(1)^{4}$, so that only 318 of the 325 singlets survive as moduli of the SCFT. The remaining seven are "eaten" by the super Higgs mechanism. It is not surprising that this is the same result as already described in [14] for the Landau-Ginzburg model.

At a generic point in the Landau-Ginzburg phase all three-point couplings are zero, for the four additional fields in the $k=5$ twisted sector do not occur. However, in our exactly solvable model there exists one coupling which can be nonzero by $\mathrm{R}$-charge conservation, namely

$$
\left\langle T^{\prime} S^{\prime} S^{\prime}\right\rangle
$$

By taking into account that every individual left and right moving $U(1)$ charge has to be conserved one finds that actually only the following three-point functions have a chance to be nonzero:

$$
\left\langle T^{\prime} S_{b}^{(i)} S_{c}^{\prime(j)}\right\rangle
$$

where $i, j=1,0,-1$ are indices of the adjoint representation of $S U(2)$. Using the explicit form of the three-point functions of the $\mathrm{N} 2 \mathrm{Vir}(\mathrm{k}=3)$ and $U(1)_{2}$ models [30], one obtains for this coupling the nonvanishing value

$$
C_{T^{\prime} S_{b}^{\prime(i)} S_{c}^{\prime(j)}}=\sqrt{3}\left(\begin{array}{ccc}
0 & 1 & 1 \\
0 & i & j
\end{array}\right) \kappa^{3}, \quad \kappa=\sqrt{\frac{\Gamma\left(\frac{3}{5}\right)^{3} \Gamma\left(\frac{1}{5}\right)}{\Gamma\left(\frac{2}{5}\right)^{3} \Gamma\left(\frac{4}{5}\right)}},
$$

where $\left(\begin{array}{ccc}j_{1} & j_{2} & j_{3} \\ m_{1} & m_{2} & m_{3}\end{array}\right)$ denotes Wigner's $3 j$ symbols. Before discussing the resulting obstruction we move forward and calculate all nonvanishing four-point couplings of the gauge singlets. $\mathrm{R}$-charge conservation tells us that there are only three possible types of such couplings

$$
\left\langle T^{\prime} T^{\prime} S S\right\rangle, \quad\left\langle T^{\prime} S^{\prime} S^{\prime} S\right\rangle, \quad\left\langle S^{\prime} S^{\prime} S^{\prime} S^{\prime}\right\rangle .
$$

The detailed analysis of all $U(1)$ charges shows that only the following three couplings of the third type in (4.23) satisfy all selection rules:

$$
\left\langle S_{a}^{\prime} S_{a}^{\prime} S_{c}^{\prime i} S_{c}^{\prime j}\right\rangle, \quad\left\langle S_{a}^{\prime} S_{b}^{\prime i} S_{b}^{\prime j} S_{c}^{\prime k}\right\rangle, \quad\left\langle S_{b}^{\prime i} S_{b}^{\prime j} S_{b}^{\prime k} S_{b}^{\prime l}\right\rangle
$$

As explicitly shown by E. Silverstein in [26], the mere fact that a four-point function does satisfy all selection rules does not guarantee that the superpotential coupling is nonzero. In [26] it was shown that the fourth order coupling of certain twisted singlets for the quintic in $\mathbb{C P}[4]$ miraculously vanishes even though the conformal field theoretic four-point function is apparently nonzero. We can follow the calculation carried out in [26] for the three fourth order couplings above (4.24), from which we discuss the first one in more detail. First, we want to calculate the four-point function

$$
\left\langle V_{-1}^{c}\left(z_{4}, \bar{z}_{4}\right) V_{0}^{c}\left(z_{3}, \bar{z}_{3}\right) V_{-\frac{1}{2}}^{a}\left(z_{2}, \bar{z}_{2}\right) V_{-\frac{1}{2}}^{a}\left(z_{1}, \bar{z}_{1}\right)\right\rangle
$$


Since in this case no contact terms can arise, we can take the zero momentum limit just from the beginning. The four vertex operators at zero momentum are

$$
\begin{aligned}
& V_{-\frac{1}{2}}^{a}\left(z_{1}, \bar{z}_{1}\right)=e^{-\frac{\rho\left(\bar{z}_{1}\right)}{2}} S^{\alpha}\left(\bar{z}_{1}\right)\left[\begin{array}{ccc}
-4 & -1 \\
3 & 1
\end{array}\right]\left[\begin{array}{ccc}
-1 & 0 \\
2 & 1
\end{array}\right]^{2}\left[\begin{array}{ccc}
-2 & -2 \\
1 & 1
\end{array}\right]^{2}[1]\left(z_{1}, \bar{z}_{1}\right),
\end{aligned}
$$

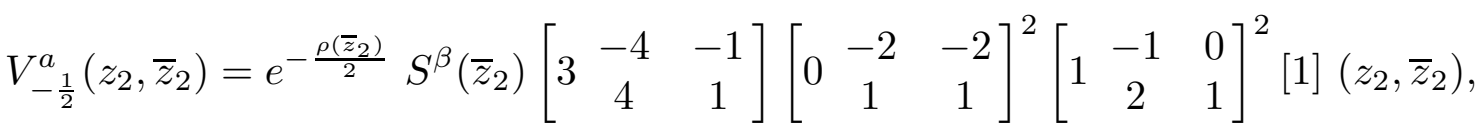

$$
\begin{aligned}
& V_{0}^{c}\left(z_{3}, \bar{z}_{3}\right)=\left[\begin{array}{ccc}
-1 & 0 \\
1 & 1 & 2
\end{array}\right]\left[\begin{array}{ccc}
1 & -1 & 0 \\
& 1 & 0
\end{array}\right]^{4}[-2]\left(z_{3}, \bar{z}_{3}\right), \\
& V_{-1}^{c}\left(z_{4}, \bar{z}_{4}\right)=e^{-\rho\left(\bar{z}_{4}\right)}\left[\begin{array}{ccc}
-1 & -2 \\
1 & 0
\end{array}\right]\left[\begin{array}{ccc}
1 & -1 & 0 \\
1 & 0
\end{array}\right]^{4}[0]\left(z_{4}, \bar{z}_{4}\right) \text {. }
\end{aligned}
$$

Using $\mathrm{N} 2 \operatorname{Vir}(\mathrm{k})=\frac{S U(2)_{k}}{U(1)} \times U(1)$ we split the primary fields of $\mathrm{N} 2 \mathrm{Vir}(\mathrm{k}=3)$ into parafermionic primaries and vertex operators of the free boson $\phi$ :

$$
\left[\begin{array}{lll}
l & q & s \\
& \bar{q} & \bar{s}
\end{array}\right](z, \bar{z})=\phi_{q-s, \bar{q}-\bar{s}}^{l}(z, \bar{z}) e^{i \alpha_{q, s} \phi(z)} e^{i \alpha_{\bar{q}, \bar{s}} \phi(\bar{z})}
$$

with $\alpha_{q, s}=\frac{1}{\sqrt{15}}\left(-q+\frac{5}{2} s\right)$. The correlation functions of the four-dimensional space-time fields and the ghost system are quite simple:

$$
\begin{aligned}
\left\langle S^{\beta}\left(\bar{z}_{2}\right) S^{\alpha}\left(\bar{z}_{1}\right)\right\rangle & =\frac{\delta_{\alpha \beta}}{\left(\bar{z}_{2}-\bar{z}_{1}\right)^{\frac{1}{2}}} \\
\left\langle e^{-\rho\left(\bar{z}_{4}\right)} e^{-\frac{\rho\left(\bar{z}_{2}\right)}{2}} e^{-\frac{\rho\left(\bar{z}_{1}\right)}{2}}\right\rangle & =\frac{1}{\left(\bar{z}_{2}-\bar{z}_{1}\right)^{\frac{1}{4}}\left(\bar{z}_{4}-\bar{z}_{1}\right)^{\frac{1}{2}}\left(\bar{z}_{4}-\bar{z}_{2}\right)^{\frac{1}{2}}} .
\end{aligned}
$$

Now, by using $S L(2, \mathbb{C})$ we set $z_{4}=0, z_{2}=1$ and $z_{1}=\infty$ and realize that the correlation functions (4.28) and the measure in (4.13) are independent of the variable $z_{3}=: x$. The correlation functions for the vertex operators in (4.27) and the $U(1)_{2}$ piece can be expressed in terms of $x$ as

$$
\langle\ldots\rangle_{U(1)}=|x|^{-\frac{4}{3}}|1-x|^{-\frac{4}{3}} .
$$

Thus, it only remains to determine five four-point functions for the parafermionic piece:

$$
\begin{aligned}
& P_{1}=\left\langle\phi_{0,0}^{0}\left(z_{4}, \bar{z}_{4}\right) \phi_{0,0}^{0}\left(z_{3}, \bar{z}_{3}\right) \phi_{-1,-1}^{1}\left(z_{2}, \bar{z}_{2}\right) \phi_{1,1}^{1}\left(z_{1}, \bar{z}_{1}\right)\right\rangle \\
& P_{2}=P_{3}=\left\langle\phi_{-1,1}^{1}\left(z_{4}, \bar{z}_{4}\right) \phi_{0,0}^{0}\left(z_{3}, \bar{z}_{3}\right) \phi_{-1,1}^{1}\left(z_{2}, \bar{z}_{2}\right) \phi_{-1,1}^{1}\left(z_{1}, \bar{z}_{1}\right)\right\rangle \\
& P_{4}=P_{5}=\left\langle\phi_{0,0}^{0}\left(z_{4}, \bar{z}_{4}\right) \phi_{-1,1}^{1}\left(z_{3}, \bar{z}_{3}\right) \phi_{-1,1}^{1}\left(z_{2}, \bar{z}_{2}\right) \phi_{-1,1}^{1}\left(z_{1}, \bar{z}_{1}\right)\right\rangle .
\end{aligned}
$$

In contrast to the four-point function in [26], here all parafermionic amplitudes can be expressed in terms of two- and three-point functions. These are well known [30], so that we arrive for the parafermionic correlation function at

$$
\prod_{i=1}^{5} P_{i}=\kappa^{4}|x|^{-\frac{4}{15}}|1-x|^{-\frac{4}{15}}
$$


Inserting $(4.29,4.31)$ into the superpotential coupling $(4.25)$, one finally obtains

$$
\begin{aligned}
\left\langle S_{c}^{\prime(i)} S_{c}^{\prime(j)} S_{a}^{\prime} S_{a}^{\prime}\right\rangle & =\sqrt{3}\left(\begin{array}{lll}
0 & 1 & 1 \\
0 & i & j
\end{array}\right) \int d^{2} x \kappa^{4}|x|^{-\frac{8}{5}}|1-x|^{-\frac{8}{5}} \\
& =\sqrt{3}\left(\begin{array}{lll}
0 & 1 & 1 \\
0 & i & j
\end{array}\right) \kappa^{4} B\left(\frac{1}{5}, \frac{1}{5}, \frac{3}{5}\right)
\end{aligned}
$$

with

$$
B(a, b, c)=\pi \frac{\Gamma(a) \Gamma(b) \Gamma(c)}{\Gamma(a+b) \Gamma(b+c) \Gamma(c+a)} .
$$

This coupling is finite and nonzero. In the same way, one obtains for the second coupling in $(4.24)$

$$
\begin{aligned}
\left\langle S_{a}^{\prime} S_{b}^{\prime(i)} S_{b}^{\prime(j)} S_{c}^{\prime(k)}\right\rangle & =-\frac{3}{\sqrt{2}}\left(\begin{array}{ccc}
1 & 1 & 1 \\
-(i+j) & i & j
\end{array}\right)\left(\begin{array}{ccc}
0 & 1 & 1 \\
0 & i+j & k
\end{array}\right) \int d^{2} x \kappa^{4}|x|^{-\frac{6}{5}}|1-x|^{-\frac{8}{5}} \\
& =-\frac{3}{\sqrt{2}}\left(\begin{array}{ccc}
1 & 1 & 1 \\
-(i+j) & i & j
\end{array}\right)\left(\begin{array}{ccc}
0 & 1 & 1 \\
0 & i+j & k
\end{array}\right) \kappa^{4} B\left(\frac{2}{5}, \frac{1}{5}, \frac{2}{5}\right) .
\end{aligned}
$$

The calculation of the most complicated third coupling fortunately is exactly the same as for the twisted fourth order coupling in (4.24) implying that it vanishes after performing the integral over the complex plane,

$$
\left\langle S_{b}^{\prime} S_{b}^{\prime} S_{b}^{\prime} S_{b}^{\prime}\right\rangle=0
$$

From the conformal field theory point of view we have yet no understanding why this happens, in particular the arguments of [27] suggest that the corresponding twisted fields for the quintic are truly moduli, so that all couplings involving this field should vanish.

\section{Consequences for the $(0,2)$ moduli space}

The few nonzero superpotential couplings calculated so far do have already interesting consequences for the moduli space. However, first we want to discuss the four singlets $T^{\prime}$. Since singlets of this kind do not occur at a general point in the Landau-Ginzburg model, it is tempting to identify them with those appearing in the $k=5$ twisted sector for our special choice of the polynomials (3.2). Hence, one would expect these fields to get a mass, when one generically deforms the complex and bundle structure. Indeed, even though we cannot calculate them exactly, there exist, for instance, sixth order couplings like

$$
\left\langle T^{\prime} T^{\prime} S_{h} S_{h} S_{h} S_{h}\right\rangle
$$

which can create a mass for the singlets $T^{\prime}$. Since already the three-point coupling (4.22) containing the singlet $T^{\prime}$ is finite, one does not expect miraculous cancellations for other couplings.

Besides the 318 untwisted moduli one expects at least 11 further flat directions from the twisted sector. Do the first two orders of the superpotential allow so many moduli? As 
the simple Higgs potential has taught us, this question is hard to answer, for surely we do not know the entire superpotential. However, let us mention the following observation: Suppose, one can find some untwisted singlets so that giving a VEV to the field $S_{c}^{\prime(0)}$ and these untwisted singlets satisfies D-flatness. Then the three-point coupling gives masses to the four fields $T^{\prime}$ and four of the twisted fields $S_{b}^{\prime}$. Furthermore, the four-point coupling (4.32) generates mass terms for the six fields $S_{a}^{\prime}$. Thus, including the Higgs effect we are left with exactly 329 massless fields, which is the number expected from the linear $\sigma-$ model. As we will show below, the field $S_{c}^{\prime(0)}$ is not exactly the Kähler modulus, but a linear combination of the fields $S^{\prime}$. But we are confident that the surviving number of 329 massless states is stable under "rotation" of $S_{c}^{(0)}$ to $R$. The complexified Kähler moduli space of the linear $\sigma$-model can be sketched like in Figure 1.
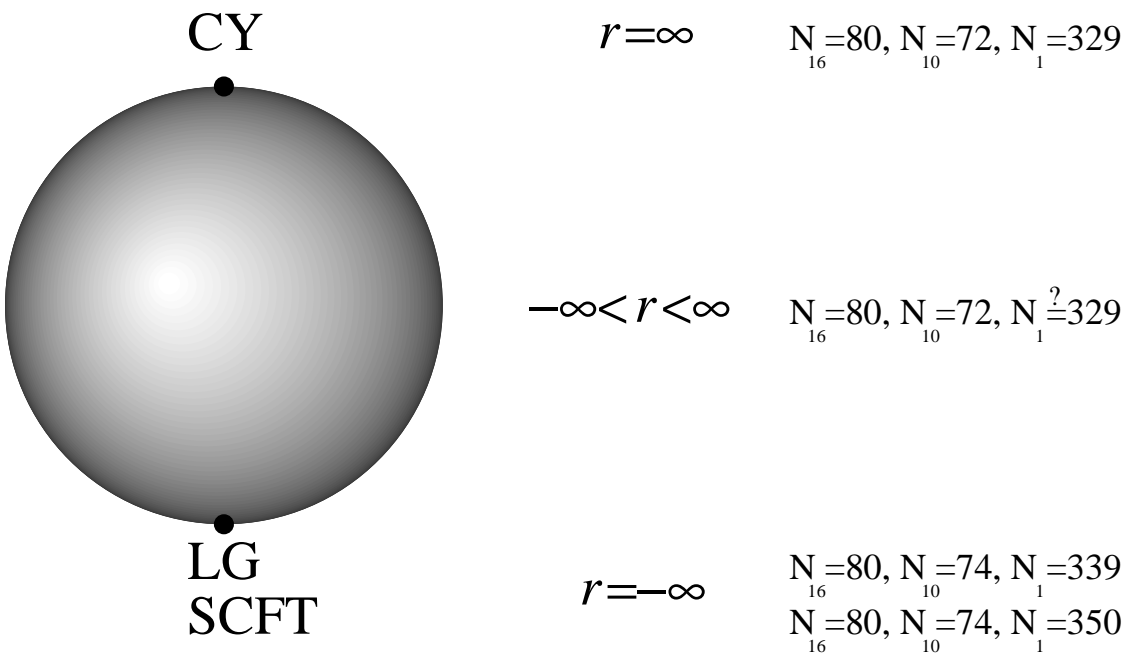

Figure 1: Kähler moduli space of the linear $\sigma$-model

In $(2,2)$ compactifications complex and Kähler moduli are related to the 27 and $\overline{27}$ matter fields by the action of the left moving supercurrent. Thus, there is an algebraic distinction among complex, Kähler and gauge bundle moduli even for small radius. In the $(0,2)$ case there exist no left moving supercurrent, so that a priori there is no way to decide to which class of moduli a given singlet belongs. In the following, we will show how to use the calculated couplings to determine the form of the Kähler modulus at least to lowest order. The following properties are expected from a modulus leading away from the LandauGinzburg radius $r=-\infty$ :

a.) We completely know the scalar potential in the renormalizable limit. Thus, in order to determine the local flat direction we require D-flatness (4.4) and F-flatness up to cubic couplings.

b.) By deforming the radius we expect to obtain the massless spectrum of the large radius CY limit. In particular, the two twisted chiral multiplets in the vector representation 
of $S O(10)$ should gain a mass.

c.) The special enhanced gauge group $S U(2) \times U(1)^{4}$ is a pure stringy effect and therefore not present in the CY phase.

d.) The massless spectrum features the permutation symmetry $S_{4}$ in the last four tensor factors. We require that the unique Kähler modulus $R$ also has this $S_{4}$ symmetry.

e.) The untwisted $S O(10)$ singlets are given by polynomials of degree four modulo some relations. This is analogous to the complex and bundle deformations of the CalabiYau manifold. Consequently, we expect $R$ to have contributions only from the $S^{\prime}$ twisted sector.

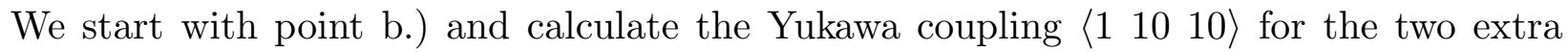
$\mathbf{1 0}^{\prime}$ s from the twisted sector. They are of the form listed in Table 5.1 in the $\left[1^{-2}\right]$ sector of $S O(8)$.

\begin{tabular}{|l|cc|c|l|l|}
\hline Type & $\mathcal{O}_{1}$ & $\mathcal{F}$ & rep. & deg. \\
\hline \hline$G$ & {$\left[\begin{array}{ccc}1 & -1 & 0 \\
& 1 & 0\end{array}\right]\left[\begin{array}{ccc}1 & -1 & 0 \\
1 & 0\end{array}\right]\left[\begin{array}{ccc}-1 & 0 \\
1 & 0\end{array}\right]\left[\begin{array}{ccc}-1 & 0 \\
1 & 0\end{array}\right]\left[\begin{array}{ccc}1 & -1 & 0 \\
1 & 0\end{array}\right][2]$} & 2 & 2 \\
\hline
\end{tabular}

Table 5.1 Twisted $\mathbf{1 0}^{\prime} \mathrm{s}$

Now, one can look for Yukawa couplings containing two twisted $\mathbf{1 0}^{\prime}$ s and one twisted singlet $S^{\prime}$. The selection rules allow only one such coupling, which can be calculated in the usual way:

$$
\left\langle S_{c}^{\prime(i)} G^{(j)} G^{(k)}\right\rangle=\frac{1}{3}\left(\begin{array}{ccc}
1 & \frac{1}{2} & \frac{1}{2} \\
-(j+k) & j & k
\end{array}\right)\left(\begin{array}{ccc}
0 & 1 & 1 \\
0 & i & j+k
\end{array}\right) \kappa^{3} .
$$

Thus, deforming in the direction of the singlet $S_{c}^{\prime}$ gives $G$ a mass and one is left with the large radius limit for the number of 10s. However, since the $S U(2)$ triplet $S_{c}^{\prime}$ alone can not break the $S U(2)$ gauge group completely, $R$ must contain contributions from other twisted states. The most general ansatz compatible with b.) - e.) is

$$
R=\sum_{j=-1}^{1} \gamma_{j} S_{c}^{\prime(j)}+\sum_{j=-1}^{1} \sum_{m=1}^{4} \beta_{j} S_{b, m}^{\prime(j)}+\sum_{n=1}^{6} \alpha S_{a, n}^{\prime}
$$

Plugging this ansatz into the flatness conditions (4.4) allows up to gauge transformations only the following two parameter solution:

$$
R=\gamma S_{c}^{\prime(0)}+\sum_{m=1}^{4} \beta\left(S_{b, m}^{\prime(-1)}+S_{b, m}^{\prime(+1)}\right)+\sum_{n=1}^{6} \frac{\gamma}{\sqrt{6}} S_{a, n}^{\prime} .
$$

Using the four-point functions (4.32-4.34), one can show that $R^{4} \neq 0$ for all $\gamma, \beta \in \mathbb{C}, \gamma \neq 0$. Since we know that there must exist a flat solution and up to first order the solution is highly restricted, we conclude that the nonvanishing four-point coupling indicates merely that the conformal fields are an unappropriate basis, in which $R$ is curved. The solution 
(5.4) merely gives the tangent vector at the SCFT point along this curve. In order to determine the next order corrections to $R$ one also has to take into account the nonflat $K\left(\phi, \phi^{*}\right)$. The picture so far obtained for the Kähler modulus is shown in Figure 2.

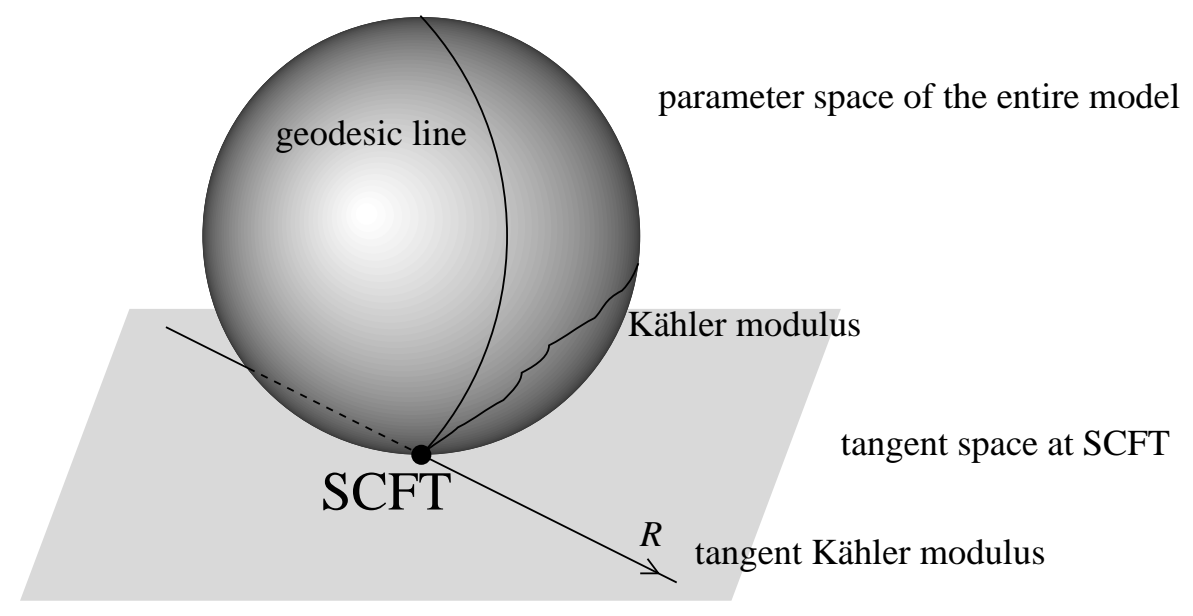

Figure 2: The Kähler modulus

The sphere visualizes the parameter space of the entire model. The superpotential is a function (or better a section) over this space. In this parameter space there is a flat direction $R$, of which our first order calculation only determines the tangent vector at the SCFT point. If the SCFT would yield appropriate coordinates (like the polar coordinates in the Mexican hat example), then the flat direction would be a geodesic line on the sphere. Knowing the singlet associated with the Kähler modulus allows one in principle to investigate couplings of the form

$$
\left\langle R^{n} 101616\right\rangle, \quad\left\langle R^{n} 11010\right\rangle .
$$

A nonvanishing coupling would detect a radius dependence of the Yukawa couplings, which perturbatively was argued to be absent $[12,18]$. Unfortunately, the selection rules do not forbid couplings of the form (5.5) and their exact calculation is hard to come by.

Since at the Landau-Ginzburg point there generically occur ten more $S O(10)$ singlets than in the Calabi-Yau phase, it is tempting to speculate about new flat directions of the superpotential leading perhaps to completely new phases of $(0,2)$ models or even to a different linear $\sigma$-model [16]. However, the data achieved so far neither rule out nor seem to prove such a possibility. We have tried to find a flat direction with a contribution from the $T^{\prime}$ field and only other twisted singlets. Since $T^{\prime}$ only appears at the very special SCFT point such a flat direction could not be part of the linear $\sigma$-model moduli space. However, for such an ansatz no solution to first order exist. If one also allows contributions from the untwisted singlets to first order there exist plenty of solutions, as for instance

$$
N=\alpha\left(\sum_{m=1}^{4}\left(S_{e, m}^{\left(\frac{1}{2}\right)}-S_{e, m}^{\left(-\frac{1}{2}\right)}\right)+\sum_{n=1}^{4} \sqrt{2}\left(S_{g, n}^{\left(\frac{1}{2}\right)}+S_{g, n}^{\left(-\frac{1}{2}\right)}\right)+\sum_{p=1}^{4} T_{p}^{\prime}\right) .
$$


Finally, we want to mention a coincidence, which might perhaps lead to a better understanding of the $(0,2)$ moduli space. The number of scalars in the vector representation of $S O(10)$ is $N_{10}=74$, which is the same as the sum of complex moduli $\left(b_{21}=73\right)$ and Kähler moduli $\left(b_{11}=1\right)$ of the underlying Calabi-Yau manifold.

\section{Summary}

In this paper we have provided further convincing arguments for the identification of an exactly solvable $(0,2)$ string model with a special point in the Landau-Ginzburg phase of a $(0,2)$ linear $\sigma$-model. Then, for the first time, we calculated exactly all three- and four-point couplings in the space-time superpotential yielding obstructions against the deformation in all 350 directions simultaneously. Similarly to the Landau-Ginzburg analysis the nontwisted moduli could be derived simply by selection rules. Furthermore, to lowest order we have up to two parameters identified the singlet corresponding to the Kähler modulus. Unfortunately, the available data did not allow us to find a unique solution for the Kähler modulus showing again the difficulty in making $(0,2)$ models technically as well treatable as $(2,2)$ models. Since we did not know the entire superpotential, we could only speculate about the possibility of further flat directions leading perhaps to another linear $\sigma-$ model.

\section{Acknowledgements}

It is a pleasure to thank L. Dolan, S. Kachru, W. Nahm, R. Schimmrigk, E. Silverstein and E. Witten for discussion. This work is supported by U.S. DOE grant No. DE-FG05$85 \mathrm{ER}-40219$.

\section{References}

[1] J.A. Bagger, Coupling the gauge invariant supersymmetric nonlinear sigma-model to supergravity, Nucl. Phys. B211 (1983) 302

[2] T. Banks, L.J. Dixon, D. Friedan and E. Martinec, Phenomenology and conformal field theory, or Can string theory predict the weak mixing angle?, Nucl. Phys. B299 (1988) 613

[3] P. Berglund, C.V. Johnson, S. Kachru and P. Zaugg, Heterotic Coset Models and $(0,2)$ String Vacua, Nucl. Phys. B460 (1996) 252, hep-th/9509170

[4] R. Blumenhagen and A. Wißkirchen, Exactly solvable $(0,2)$ supersymmetric string vacua with GUT gauge groups, Nucl. Phys. B454 (1995) 561, hep-th/9506104

[5] R. Blumenhagen, A. Wißkirchen and R. Schimmrigk, The $(0,2)$ exactly solvable structure of chiral rings, Landau-Ginzburg theories and Calabi-Yau manifolds, Nucl. Phys. B461 (1996) 460, hep-th/9510055

[6] R. Blumenhagen and A. Wißkirchen, Exactly solvable points in the moduli space of heterotic $N=2$ strings, preprint IFP-606-UNC, BONN-TH-96-01, hep-th/9601050

[7] P. Candelas, G.T. Horowitz, A. Strominger and E. Witten, Vacuum configurations for superstrings, Nucl. Phys. B258 (1985) 46

[8] P. Candelas and X. De la Ossa, Moduli space of Calabi-Yau manifolds, Nucl. Phys. B355 (1991) 455 
[9] E. Cremmer, S. Ferrara, L. Girardello, B. Julia, J. Scherk and P. van Nieuwenhuizen, Spontaneous symmetry breaking and Higgs effect in supergravity without cosmological constant, Nucl. Phys. B147 (1979) 105

[10] M. Dine, N. Seiberg, X.G. Wen and E. Witten, Nonperturbative effects on the string world sheet I+II, Nucl. Phys. B278 (1986) 769, ibid. B289 (1987) 319

[11] J. Distler and B. Greene, Aspects of $(2,0)$ string compactifications, Nucl. Phys. B304 (1988) 1

[12] J. Distler and B. Greene, Some exact results on the superpotential from Calabi-Yau compactifications, Nucl. Phys. B309 (1988) 295

[13] J. Distler and S. Kachru, (0,2) Landau-Ginzburg theory, Nucl. Phys. B413 (1994) 213, hep-th/9309110

[14] J. Distler and S. Kachru, Singlet couplings and (0,2) models, Nucl. Phys. B430 (1994) 13, hep-th/9406090

[15] J. Distler and S. Kachru, Quantum symmetries and stringy instantons, Phys. Lett. B336 (1994) 368, hep-th/9406091

[16] J. Distler and S. Kachru, Duality of (0,2) string vacua, Nucl. Phys. B442 (1995) 64, hep-th/9501111

[17] D. Gepner, Yukawa couplings for Calabi-Yau string compactifications, Nucl. Phys. B311 (1988) 191

[18] B.R. Greene, Superconformal compactifications in weighted projective space, Commun. Math. Phys. 130 (1990) 335

[19] K. Intriligator, Bonus symmetry in conformal field theory, Nucl. Phys. B332 (1990) 541

[20] S. Kachru and E. Witten, Computing the complete massless spectrum of a LandauGinzburg orbifold, Nucl. Phys. B407 (1993) 637, hep-th/9307038

[21] T. Kawai and K. Mohri, Geometry of $(0,2)$ Landau-Ginzburg orbifolds, Nucl. Phys. B425 (1994) 191, hep-th/9402148

[22] A.N. Schellekens and S. Yankielowicz, Extended chiral algebras and modular invariant partition functions, Nucl. Phys. B327 (1989) 673

[23] A.N. Schellekens and S. Yankielowicz, Modular invariants from simple currents. An explicit proof, Phys. Lett. B227 (1989) 387

[24] A.N. Schellekens and S. Yankielowicz, New modular invariants for $N=2$ tensor products and four-dimensional strings, Nucl. Phys. B330 (1990) 103

[25] A.N. Schellekens and S. Yankielowicz, Simple currents, modular invariants and fixed points, Int. J. Mod. Phys. A5 (1990) 2903

[26] E. Silverstein, Miracle at the Gepner point, Phys.Lett.B352(1995)69, hep-th/9503150

[27] E. Silverstein and E. Witten, Criteria for conformal invariance of $(0,2)$ models, Nucl. Phys. B444 (1995) 161, hep-th/9503212

[28] E. Witten, New issues in manifolds of SU(3) holonomy, Nucl. Phys. B268 (1986) 79

[29] E. Witten, Phases of $N=2$ theories in two dimensions, Nucl. Phys. B403 (1993) 159, hep-th/9301042

[30] A.B. Zamolodchikov and V.A. Fateev, Parafermionic currents in two-dimensional conformal quantum field theory and selfdual critical points in $Z(N)$ symmetric statistical systems, Sov. Phys. JETP 62 (1985) 215 